\title{
Compression and Visualization Interactive of 3D mesh
}

\author{
Zeineb Abderrahim \\ UR-SETIT, ISBS, 3038 \\ Sfax, Tunisia \\ zeineb.abderrahim@yahoo.fr
}

\author{
Mohamed Salim Bouhlel \\ UR-SETIT, ISBS, 3038 \\ Sfax, Tunisia \\ medsalim.bouhlel@enis.rnu.tn
}

Received: April 10, 2019. Revised: May 25, 2021. Accepted: October 23, 2021. Published: November 16, 2021.

\begin{abstract}
The combination of compression and visualization is mentioned as perspective, very few articles treat with this problem. Indeed, in this paper, we proposed a new approach to multiresolution visualization based on a combination of segmentation and multiresolution mesh compression. For this, we proposed a new segmentation method that benefits the organization of faces of the mesh followed by a progressive local compression of regions of mesh to ensure the refinement local of the three-dimensional object. Thus, the quantization precision is adapted to each vertex during the encoding /decoding process to optimize the rate-distortion compromise. The optimization of the treated mesh geometry improves the approximation quality and the compression ratio at each level of resolution. The experimental results show that the proposed algorithm gives competitive results compared to the previous works dealing with the rate-distortion compromise and very satisfactory visual results.
\end{abstract}

Keywords-3D mesh; Visualization interactive; $3 D$ segmentation; Progressive compression; Refinement local

\section{INTRODUCTION}

Thanks to the increase of computer performance (computing capacity, memory and graphics) and advanced Internet networks, the three-dimensional objects are increasingly becoming used in our daily lives and in many fields including computer-aided design (CAD), scientific simulation, augmented reality, virtual visits, medicine, video games or movies. These 3D objects are usually presented in the form of a triangular surface mesh due to their efficiency and simplicity: the geometric structure is mainly composed of geometry and connectivity. In other words, geometry presents the vertex positions, but connectivity presents the way how the vertices are connected together to form triangles.

Recent technological developments in various areas have given birth to different techniques that generate meshes with sizes very large and voluminous so that it becomes difficult to load in all their view, and for transmitting on the network. Therefore, for facilitating the visualization of this type of 3D object, it was necessary to use compression techniques ensuring progressive multiresolution representation of such an object, and segmentation methods that facilitate selective refinement of a part thereof.

In this context, the progressive compression methods are very effective because they offer the ability to visualize, compress and progressively transmit the object at different levels of detail. Therefore, these methods allow the adaptation transfer of these objects to different client resources (rate of networks, visualization capacity of the terminal). For this reason, we have presented a progressive compression method based on multi-resolution analysis which focuses on irregular meshes using an adaptation of quantization precision for each mesh vertex to optimize rate-distortion compromise.

Indeed, our work has as objective to propose a method of visualization interactive of multiresolution significant parts of the $3 \mathrm{D}$ object based on a combination of a progressive compression scheme and segmentation technique of triangular mesh surfacique. However, this approach has led to distortions (appearance of holes) for the boundaries between the different regions significant. The solution we proposed based on KNN classifier (K-the Nearest Neighbor), which moving the vertex of the subdivided area to the nearest vertex in the nonsubdivided area.

This article consists of three parts. In the first part, we present the principal existing work of progressive compression and visualization of $3 \mathrm{D}$ mesh. In the second part, we present the basic algorithm used and our method of improvement of the rate - distortion and then we describe the process of interactive visualization. The last part is devoted to show the results obtained with our proposal, comparing with some methods presented in the previous section, followed by the conclusion.

\section{THE PREVIOUS WORK}

\section{A. The progressive compression}

The progressive compression methods rested on a coarse mesh and refinement information gradually transmitted to obtain different levels of mono-resolution. Unlike the monoresolution methods, they offer the possibility of accessing the intermediate reconstructions of a $3 \mathrm{D}$ object during its transmission.

The first progressive compression method was introduced by Hoppe [13] which consists in iteratively applying an operation edge contraction "edge collapse" during the encoding to generate a coarse mesh. The reconstruction is done by a reverse operation "vertex split" while decoding. The total cost is non-linear $(n \cdot \log (n))$; it is reserved for meshes of low complexity. Cohen-Or and al [7] propose a simplification 
algorithm based on the technique of color patches and the successive withdrawals of vertices followed by deterministic re-triangulation. These patches are colored by using 2 or 4 colors to allow the decoder to properly insert vertices in the reconstruction based on the color patches. This algorithm compresses connectivity with a cost of 6 bits/vertex.

The previous algorithm was recently improved by Alliez and Desbrun [1] who introduced an algorithm based on the progressive deletion of independent vertices with a retriangulation step under the constraint of maintaining the vertex degrees around 6. It has an average coding cost of 1.85 bits/face. The conservation of the regularity ensures an efficient connectivity coding and the connectivity coding cost is around 3.7 bits/vertex. All these approaches are directed by the connectivity as they give priority to connectivity coding. For this, the most recent progressive encoders are not only motivated by the refinement of connectivity, but also by the geometrical measurements of the rebuilt mesh.

Gandoin and Devillers [10] propose a compression algorithm which is no longer driven by connectivity but by the geometry based on the kd-tree subdivision. The main objective in their approach is to achieve continuity between the coarse approximations and the finest ones of the object. The connectivity is encoded by encoding all the change in the cell subdivision. In terms of compression rate, this algorithm has better results than the algorithms guided by connectivity. This method is improved by Peng and Kuo [25] by replacing kd-tree with Octree data structure. The Octree cells are refined in order of priority, where subdivisions of cells with the greatest improvement of distortion are executed the first. By optimizing the coding of connectivity and geometry through effective predictions, this approach gives good results for lossless compression and improved from 10 to $20 \%$ compared to [10].

Unlike the previous approaches, the spectral approach introduced by Karni and Gotsman [15] proposed a spectral decomposition by projecting the mesh geometry on a set of eigenvector from the diagonalization of the discrete Laplacian operator. This approach is generally effective for low compression rate of smooth geometry models. This results in average costs of coding around 4.5 bits / vertex. For this, these methods do not offer a progressive geometry; connectivity remains unchanged during the transmission. Indeed, most works are directed towards multi-resolution analysis which allows having both geometric and topological scalability.

At last, we can distinguish those which were focused on semi-regular meshes based on remeshing and those which were adapted to the topological structure of the irregular mesh. The pioneer progressive compression method of the semi-regular mesh was proposed in 2000 by Khodakovsky et al [16]. It consists in remeshing an arbitrary mesh $\mathrm{M}$ into a semi-regular mesh by using the MAPS algorithm (Multi-resolution Adaptive Parameterization of Surfaces) [17]. After this remeshing step, Khodakovsky et al choose to apply a transformed semi-regular wavelet based on the loop interpolating filter.

A recent method has been proposed by Payan et al [24] which is the compression algorithm with loss for dense triangular meshes incorporating a binary-allocation. This method is based on a stage of remeshing using a normal remesher and one transformed into a non lifted Butterfly wavelet.

For this, other existing techniques directly consider the irregular structure of the mesh such as the Wavemesh [27] which proposed a new approach guided by connectivity and extended the wavelet decomposition scheme on an irregular mesh so as to generate approximations of better quality than that based on geometry. The connectivity coding cost is 2 bits / vertex on average.

In 2009, Valette et al [26] proposed a new lossless progressive compression algorithm of mesh based on a metric "Incremental Parametric Refinement" named "IPR", where connectivity is not controlled in a first step, giving visually pleasant meshes to each level of resolution while saving connectivity information compared to the previous approaches. This approach shows its effectiveness in terms of ratedistortion, especially at low rate.

To optimize the rate-distortion compromise for meshes during transmission, optimization techniques are also used. The algorithm [24] optimizes the accuracy of quantization of the wavelet coefficients of each sub-frequency band so as to minimize the geometric error for a given rate. Thus, the binary allocation used to control the visual quality of the reconstructed mesh while encoding the geometry to optimize the ratedistortion compromises [23] regardless of the desired compression rate. In this context, [26] presents an approach that adapts the quantization precision of each vertex based on the local geometric configuration. This method gives good results especially at low rate.

More recently, [19] [20] presents a new method for optimizing the rate-distortion based on the adaptation of the quantization precision of geometry and color for each intermediate mesh. The used adaptation, which is performed at each iteration and is chosen from the decimation operation and the operation of decreasing the quantization precision, highly improves the rate-distortion trade-off.

\section{B. Combination of compression and visualization}

This approach is based on a combination of the segmentation of the mesh into a set of zones, named regions or cluster and the compression. Indeed, the author applies a segmentation algorithm of the mesh in the first place. Then, it applies a progressive compression method for each region separately. The random access compression schemes are called "out-of-core" enable interactive visualization of large meshes, because only the information necessary and sufficient for parts requested at a given moment are loaded into memory. These approaches often start by dividing the mesh into clusters, compress independently and allow decompression independently.

Among these approaches, Lindstrom [21] has developed a method for meshes with connectivity. An octree is used to divide the triangles into clusters and to build a multi-resolution 


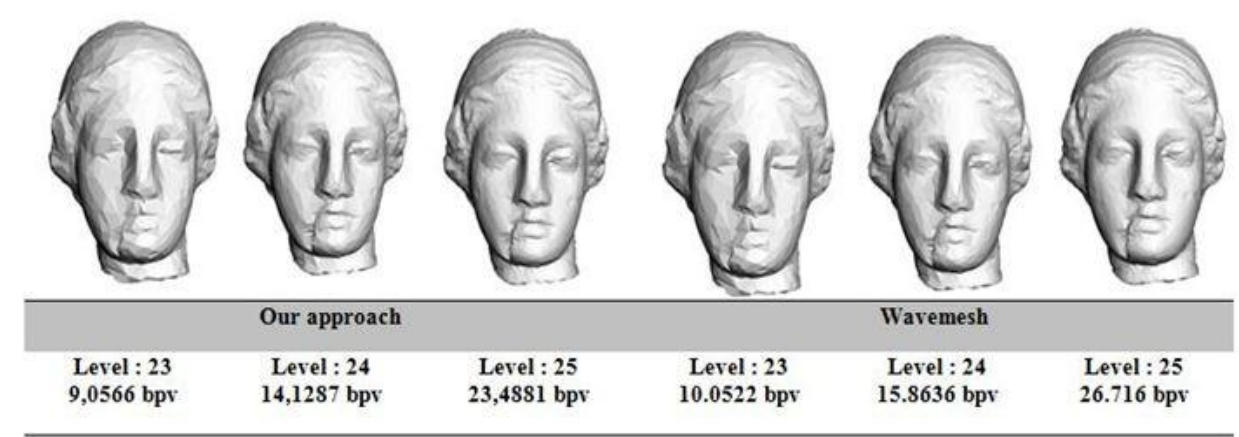

Fig. 1. Compression results of our approach and the basic algorithm Wavemesh [27] for the object Venushead at different rates.

hierarchy. A measure of quadratic error is used to select the positions of representative points for each level of detail and the refinement is guided by the visibility and the measurement of error in screen space.

Subsequently, Yoon et al. [29] proposed a similar algorithm with a bounded memory footprint: a hierarchy of clusters is constructed, each containing a progressive sub-mesh to smooth the transition between levels of detail. In this context, Cignoni et al 2003 [5] describes an efficient technique for out-of-core rendering and management of large textured terrain surfaces. The technique, called batch dynamic adaptive meshes (BDAM), is based on a tree structure associated (a quadtree). The objective is to propose a technique which is able to manage huge datasets of textured terrain without weigh down the CPU and fully exploit the power of current and future graphics cards.

In 2005, Gobbetti et Marton [12] have introduced fars voxels, capable of displaying both regular mesh that a soups of triangles. The principle is to transform the volume sub-parts of the model in approximations of their appearance at a certain distance, following the angle of view. In 2008, Clment Jamin et al [14] the method proposed out-of-core that combines good performance in compression and interactive visualization. They chose as a starting point the compression algorithm in-core without loss of Gandoin and Devillers [11]. The basic idea is to divide the original object in a tree mesh independent by introducing a hierarchical primary structure (nSP-tree). In fact, this method reduces the size of objects while allowing interactive navigation in real time.

In addition, Clement Courbet et Celine Hudelot [8], have proposed a new hierarchical representation of the mesh using an approach based on a recursive division of the mesh into two independent parts, under the constraint that one of the two parties which result must be reconstructible independently. Thus, their approach decompresses gradually the requested parts of a mesh without decoding the less interesting parts. More recently, Adrien Maglo et al [22], introduces a new compression scheme randomly accessible of triangular meshes. The scheme is aimed at interactive visualization of large meshes, supporting the encoding of vertex colors. They extended the approach of [4] using the encoder progressive [18] to compress the faces of clusters. These schemas have been modified to avoid duplication of information on the geometry of the boundary between the vertices of cluster.

\section{OVERVIEW OF OUR METHOD}

Our work is a combination of a new method of segmentation and progressive compression method which aims to visualization interactive. We have chosen the method Wavemesh proposed by Valette and Prost in 2004 [27], which is an algorithm guided by connectivity. This approach, indeed, is able to directly adapt to the topological structure of irregular meshes offering new irregular subdivision schemes. The goal is to overcome the difficulty of connectivity subdivision imposed by the method of Lounsbery and maintain the geometry and connectivity of the approximations as close as possible to the original mesh. Additionally, this approach avoids the remeshing process that overcomes the difficulties of its implementation for some mesh from medical imaging and that presents an additional gain.

\section{A. Progressive compression of $3 D$ objects with an adaptive quantization}

The quantization precision is an important factor used to optimize the rate distortion. Optimization is often neglected in most researches on progressive compression. Thus, most of the existing methods use a 12 bit-scalar-uniform quantization and some approach gets around an adaptation of quantization on each level of detail. In addition, we notice that the quantization precision is larger than necessary for intermediate meshes at low resolution for algorithms guided by connectivity that retain high precision of the initial quantization throughout the simplification [26]. Indeed, to optimize the rate-distortion compromise, our progressive compression method is based on the adaptive quantization algorithm IPR [26] that uses a technique of adaptive quantization on each vertex. The quantization algorithm applied at this stage consists in quantifying each vertex with a specific quantization precision. Indeed, the quantization precision is the number of bits used for quantization and it is determined according to the following procedure: The first step is to calculate for each vertex $S_{i}$ its closest neighbor, let us say the vertex $S_{j}$. Then quantization the coordinates of these vertices $S_{i}$ and $S_{j}$ using the following equation:

$$
\tilde{c}_{i}=\left\lfloor 2^{Q_{i}-Q_{m}}{ }_{i}\right\rfloor
$$


$Q_{i}$ and $Q_{m}$ respectively represent the initial quantization precision of $S_{i}$ and the value of the maximum precision such as $3<Q_{i}<Q_{m}$ with $Q_{m}=12$ while $c_{i}$ represents the coordinate of a given vertex $S_{i}$.

To determine whether the quantization precision associated with the vertex $S_{i}$ is sufficient or not, the quantization algorithms have to compute the squared distance between the two vertices $S_{i}$ and $S_{j}$ in its quantified version. This is done by the following formula:

$$
D_{i}=\left\|\tilde{c}_{i}-\tilde{c}_{j}\right\|^{2}
$$

The sufficient precision is then achieved only if the value of the distance $D_{i}$ exceeds a threshold $\mathrm{q}_{\mathrm{t}}$. According to the experiment that we carried out, we choose use the threshold value which seems to be the most appropriate and equal to 200. In the case that the quantization precision is not sufficient, we increment it with one bit and we keep iterating until the result of quantization precision is sufficient.

This technique of adaptive quantization can be summarized by the following algorithm:

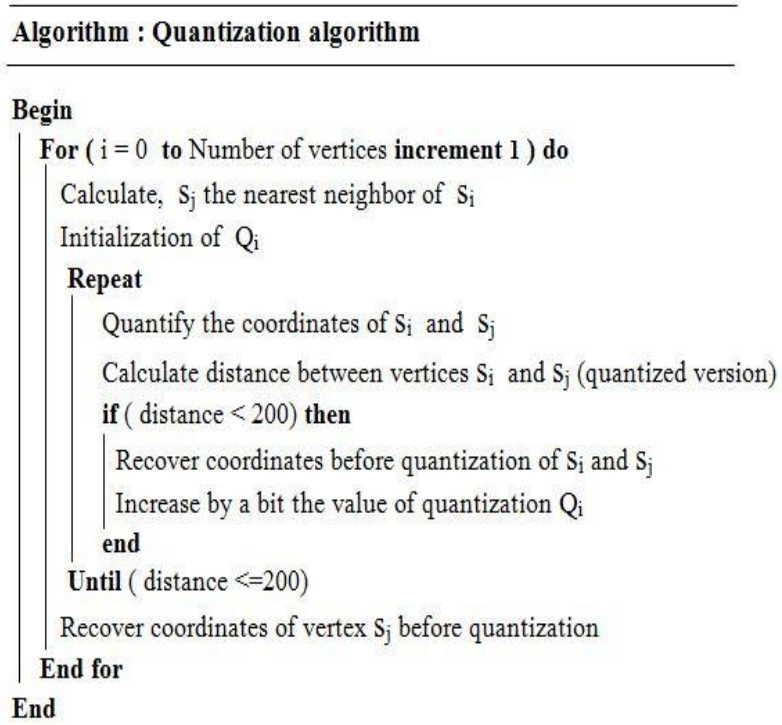

Fig. 2. Quantization algorithm of our approach

The quantified wavelet coefficients are compressed by means of adaptive arithmetic encoder because of its simplicity of use. This type of encoder provides transparent management of the probability table used for entropy coding, whose the probability table is built automatically by the encoder and decoder. At the end of decompression, we recovered the original quantization precision of the vertices.

Fig. 3 shows the Fandisk mesh model compressed with different quantization precisions. When the initial quantization $Q_{i}$ is equal to 19 bits in Fig. 3 (b), there is a significant degradation in the visual quality and a decrease in the file size. In contrast, in Fig. 3 (c), the initial quantization $Q_{i}$ is set at 20. It generates a similar geometric distortion with good visual quality but increases the size of the com-pressed file. We note that the quantization influences the size of the compressed file denoted $\mathrm{T}$, thus the adapting of quantization to complexity of intermediate meshes (number of vertices) can improve the rate-distortion compromise.

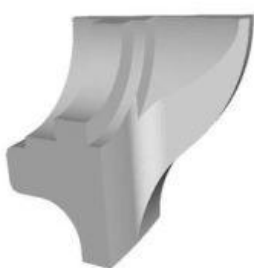

(a)

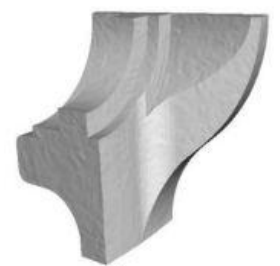

(c)

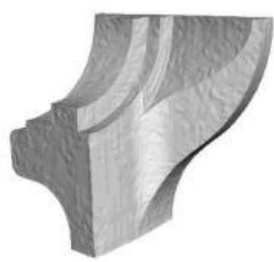

(b) (a) Original

$V: 6475$

Te $: 385$ Ko

(b) $\mathrm{Qi}=19$

$T=12,11$ bits/vertex

Tc $=9805$ bytes

(c) $\mathrm{Qi}=\mathbf{2 0}$

$\mathrm{T}=\mathbf{1 4 . 3 4} \mathrm{bits} / \mathrm{vertex}$

$\mathrm{Tc}=11609$ bytes

Fig. 3. Comparison of the file size of the original and the quantified Fandisk version by using different quantization precisions. With Qi: initial quantization, $\mathrm{T}$ : total data $(\mathrm{C}+\mathrm{G})$ and $\mathrm{Tc}$ : compressed file size.

\section{B. Visualization interactive}

1) Description of segmentation method: Our proposed method of segmentation fits into the family of segmentation surface patch designed to have significant parts. Indeed the algorithm begins with a round of the topology of the mesh. The total number of faces of the mesh will be divided by four. For each iteration, the algorithm will recover all faces with an identifier id as (i-1) * $\mathrm{n}<\mathrm{id}<\mathrm{i} * \mathrm{n}$ with $\mathrm{i}$ represents the number of the region and $\mathrm{n}$ represents the number of faces by region $n=$ nbface / 4 (such as nbface is the number of faces of the mesh in question). Then, the algorithm will recover all the vertices of these faces with their connectivity and saves them in a file that represents the corresponding region $\mathrm{i}$.

After creating a new mesh from each region, the files representing these regions will be used later in the process of visualization.

2) Interaction with the camera: In this section, we addressed the interaction of the user with the 3D object (zoom in, zoom out) depending on its position relative to the camera. 


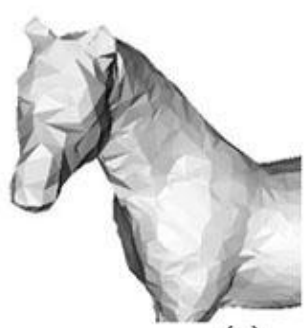

(a)

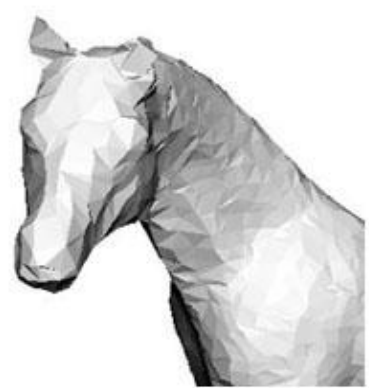

(b)

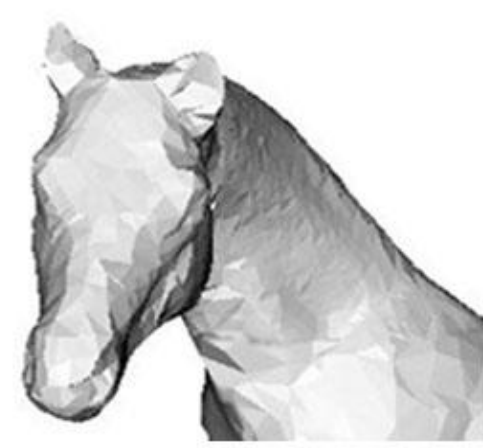

(c)

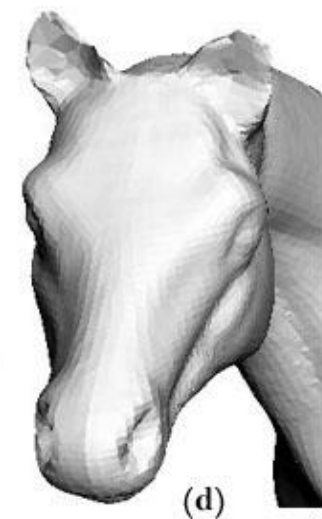

(d)

Fig. 4. Visualization interactive of horse model (Time refinement: 1,5 s from (a) to (b); 1,75 s from (b) to (c); $2,15 \mathrm{~s}$ from (c) to (d) )

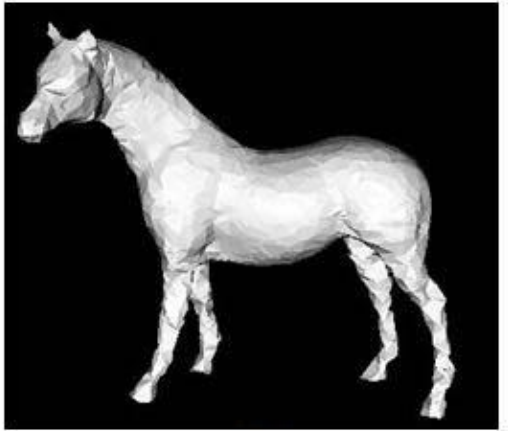

(a)

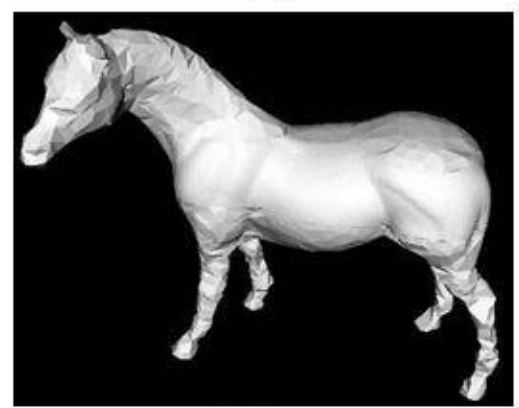

(c)

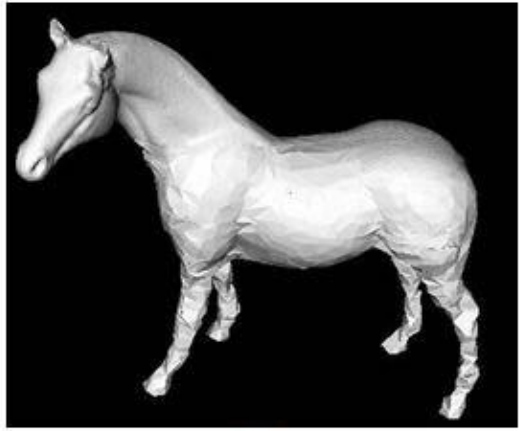

(b)

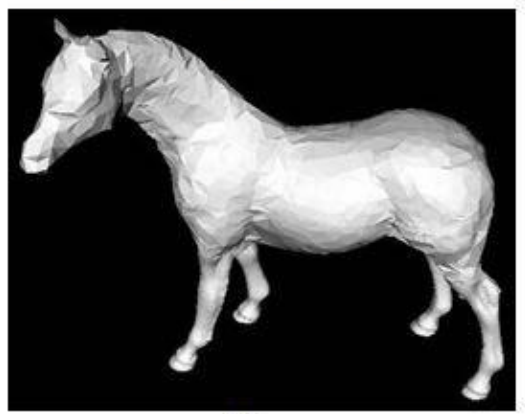

(d)

Fig. 5. Multiresolution visualization interactive of horse model.

To ensure a adaptive refinement interactive of $3 \mathrm{D}$ object, the resolution levels are adjusted according to the distance from the object to the camera and its projection on the screen. Whenever the object is closer to camera (the distance between the object and camera decreases), we augment the level of resolution of the object to make it more detailed (refined) to the user (see "Fig. 4").

In fact, on each user interaction, we calculate the more appropriate resolution of each region based on its position relative to the camera and the distance Bounds (db) that represents the size of the object. Second, the resolution of each region will be updated in the corresponding mesh depending on user interaction. Thus we have limited the number of intermediate mesh (number of filters) of each region according to the current resolution. It should be noted that we must always test the current level of precision compared to the maximum level of detail of the region, to avoid the crash of the algorithm.

Thus, Figure 5(a) shows the initial mesh (coarse) that will be refined. Figure 5(b) shows the refinement has been the head by three levels of successive refinements. The refinement in Figure 5(c) affects only the back region; other regions keep their coarse levels. Figure 5(d) shows the result of refinement of feet region by two successive levels of details. 
TABLE I. COMPARISON OF THE COMPRESSION-RATES OF GEOMETRIES

\begin{tabular}{|c|c|c|c|c|}
\hline Model & Vertices & $\begin{array}{c}\text { Wavemesh } \\
(\mathbf{1 2} \text { bits })\end{array}$ & $\begin{array}{c}\text { Our approach } \\
(\mathbf{1 0} \text { bits })\end{array}$ \\
\hline Fandisk+WGC & 6475 & 14.70 & 8.84 & 12.34 \\
\hline Fandisk & 6475 & 15.71 & 9.55 & - \\
\hline Nefertiti+WGC & 299 & 30.65 & 22.04 & 21.69 \\
\hline Nefertiti & 299 & 30.52 & 11.88 & - \\
\hline Venusbody & 711 & 26.87 & 25.50 & - \\
\hline
\end{tabular}
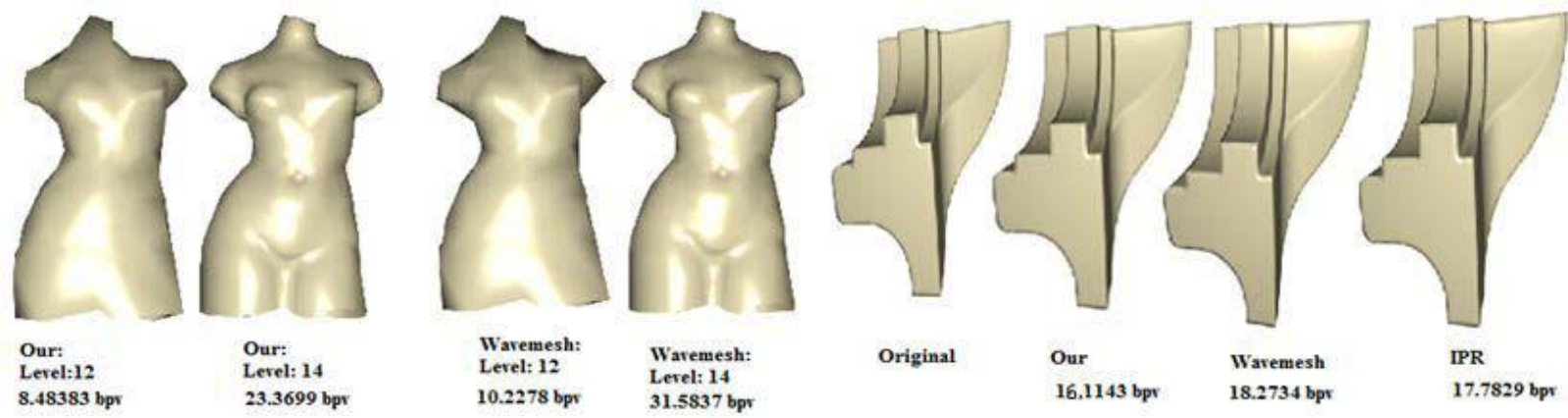

Fig. 6. Left: comparison of visual extract of intermediate meshes for Venusbody. Right: Comparison of Fandisk model with our algorithm, Wavemesh [27] and IPR [26].

3) Handling of multiresolution boundaries: Since it is possible to have two regions that do not have the same level of resolution, visual artifacts (holes) may occur. The following figure illustrates this phenomenon:

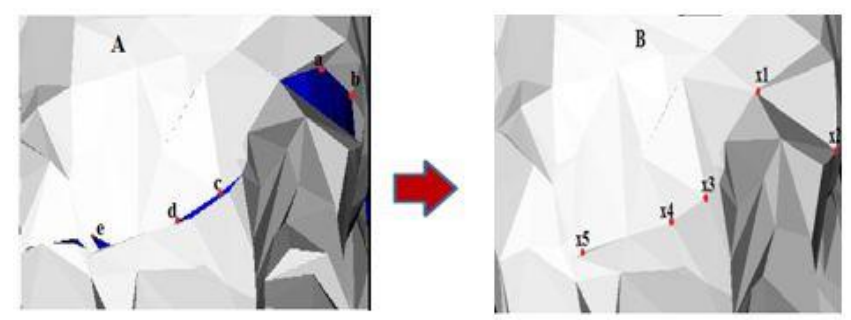

Fig. 7. Example of holes (blue areas in A) on the boundaries of two regions with two levels of different resolution.

The idea was to use the KNN (K-Nearest Neighbor) algorithm for moving the vertex of the subdivided area toward the nearest vertex in the non-subdivided area. This correction allowed us to have a better performing visualization of the 3D object. The algorithm is as follows: Recovering the vertices of borders in the region in question and its neighbors. For each point of the boundary of the region more detailed, find the nearest point which lies on the border of its neighbor, using the KNN algorithm. Subsequently, we change the coordinates of the vertex in question by the coordinates of its nearest neighbor. The Points $\mathrm{a}, \mathrm{b}, \mathrm{c}, \mathrm{d}$ and $\mathrm{e}$ in Figure $\mathrm{A}$ will be moved respectively to the points $\mathrm{x} 1, \mathrm{x} 2, \mathrm{x} 3, \mathrm{x} 4$ and $\mathrm{x} 5$ in Figure B.

\section{EXPERIMENTAL RESULTS}

\section{A. Evaluation of Compression method}

Table 1 lists the compression rate of geometry of our proposal compared with that of Valette and Prost [27] and AD [1]. On both models Fandisk and Nefertiti, we presented two results. Indeed, the first result indicates the implementation by considering the condition WGC which tends to improve the rate-distortion efficiency of the compression algorithm and the second result is without WGC.

When comparing the results obtained with and without WGC, we notice that the WGC improves the compression rate of geometry for the Fandisk model about 0.7 bit/vertex. On the other hand, for Nefertiti model, the result is less effective because the WGC does not work properly on smooth meshes. The proposed compression scheme leads to better geometric compression results than the one presented by the basic algorithm. It provides a compression gain ranging from $12 \%$ to $70 \%$. We note that at low rate the performance of our method outperforms the Wavemesh with and without the condition WGC.

Fig. 1 shows the compression results of our approach and the basic algorithm Wavemesh for the object Venushead at different rates. Thus, Fig.6 presents the visual extracts of different resolution levels of the Venusbody object respectively generated by our algorithm and the Wavemesh algorithm. Also, it presents the visual extracts of the highest 
level of Fandisk mesh resolution respectively provided by our algorithm, the Wavemesh and IPR.

We note that our approach retains a good visual quality all along the mesh refinement process by minimizing the size of the file.

Figure 8 and Figure 9 respectively show the rate-distortion curve of Fandisk and Mannequin models. Both models are quantified to 12 bits for the Wavemesh algorithm and to a quantization precision between 4 and 12 bits for our proposal. In these figures, the vertical axe indicates the amount of distortion which is the maximum RMS (root mean square) distance normalized by the diagonal of the bounding box. The RMS value is measured by the Metro tool [6] while the horizontal axis shows the rate in bit per vertex. These curves show that our algorithm optimizes the rate-distortion especially at low rate.

However, for rate-rates below $10 \mathrm{bps}$ our approach leads to better results. On the other hand, for rates higher than $10 \mathrm{bps}$, the visual quality of the generated meshes becomes almost constant and the encoders of the Wavemesh and IPR provide slightly higher rate-distortion reports. Indeed, this approach proves its high efficiency at low rate rates.

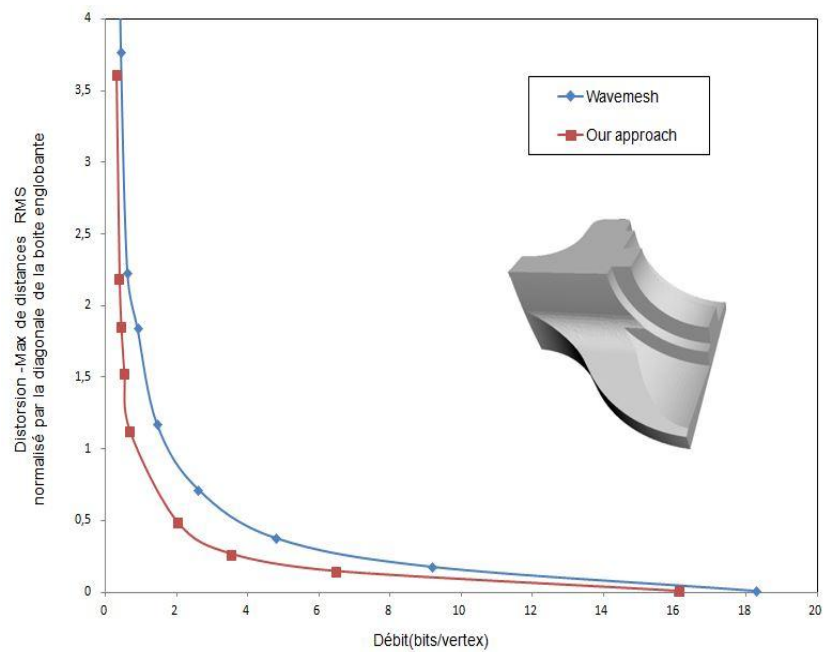

Fig. 8. Rate-distortion curve of Fandisk.

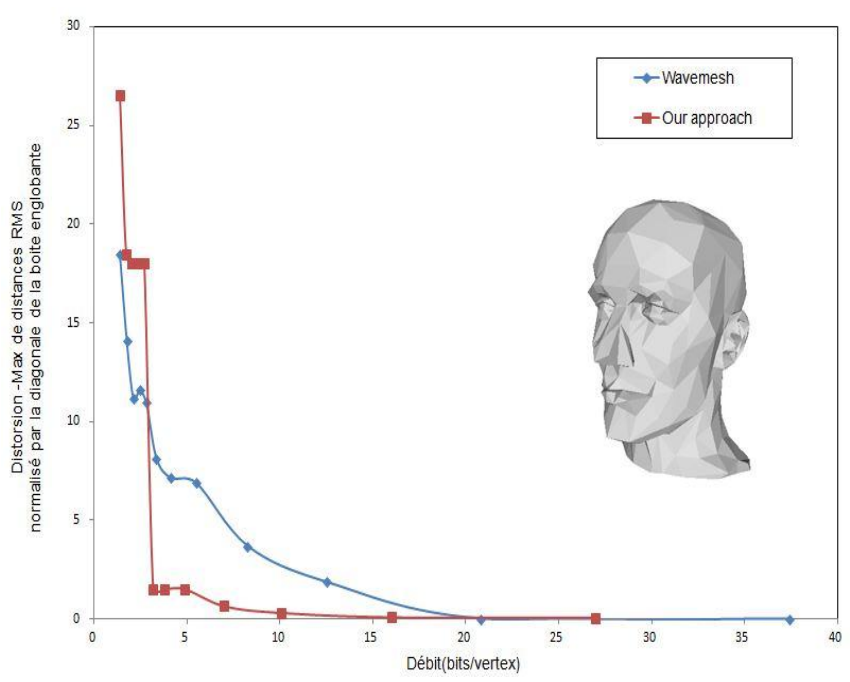

Fig. 9. Rate-distortion curve of Mannequin.

\section{B. Evaluation of Segmentation method}

The evaluation of the segmentation of a mesh is not obvious and is difficult to measure as outlined Attene et al. [2] in their comparative study of mesh segmentation. To measure the performance of our method, we use the evaluations criteria listed in [9] which are: the creation of regions and borders correct and of complexity. In addition to better demonstrate the performance of our method we compare it with other methods such as the method of 3D mesh hierarchical segmentation from dynamic contour [3] and the method of Wang Jun [28].

Figure 10 shows that our method respects well the criterion of border regions creation a more adequate than Delest and same for Wang for this object. Indeed, it seems as in figure 10 (b) and (c) there are regions whose boundaries are overlapped. By against, our method defines the boundaries more precisely. Since our method will be used for visualization and multiresolution interactive $3 \mathrm{D}$ objects it is required to reduce the complexity of the algorithm. We use the computation time of the algorithm to valorize its complexity.

Table 2 below lists the computation time of our algorithm compared with the method of Delest and Wang, in seconds:

TABLE II. COMPARISON OF EXECUTION TIMES OF THE DIFFERENT ALGORITHMS.

\begin{tabular}{|c|c|c|c|c|}
\hline Model & Vertices & Delest & Wang & Our method \\
\hline Fandisk & 6475 & $3,5^{* *}$ & $14,5^{* *}$ & 0,331 \\
\hline car & 342693 & - & $109,660^{* *}$ & 0,443 \\
\hline Horse & 48485 & - & - & 0,38 \\
\hline
\end{tabular}

Observation of the results provided in this table shows that our method has a running time of very small compared to that of Delest and Wang. The gain is presented in the order of $45 \%$ for the object Fandisk. In addition, for the object of car (car), the execution time of our segmentation algorithm is inconsiderable compared to the method of Wang.

\section{CONCLUSION}

We propose in this paper a new multiresolution interactive visualization method that allows you to locally refine the mesh in significant regions with movement of the camera, which allows to easily view the details as different parts of the object according to the user need. Our approach is based on a method for progressive compression of meshes based on the adaptation of the quantization precision of geometry. Thus, we have proposed a new segmentation method which refers to neighborly relations and connectivity between the faces.

The experimental results show that this method of adaptation of quantization produces very satisfactory visual results while minimizing the size of file. Thus, our segmentation approach provides a good characterization boundary with a speed of calculation. In addition, we solved the problem of holes between regions. 

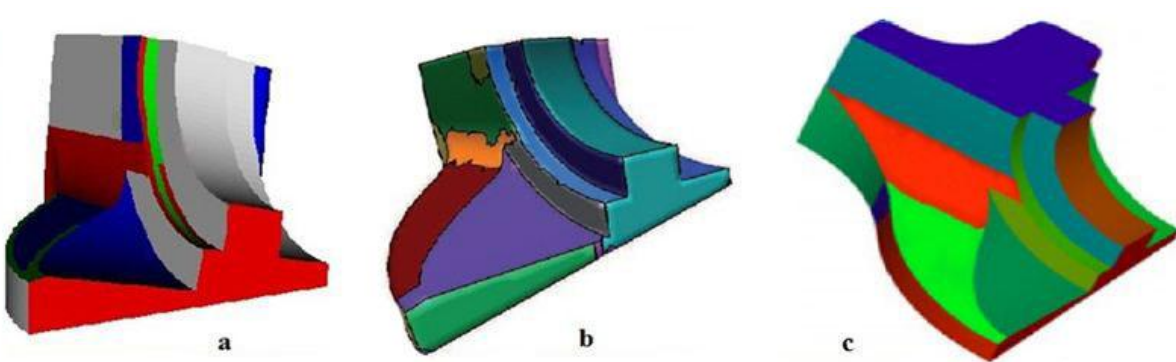

Fig. 10. Comparison of creating borders: (a) our method, (b) the method of Deleste (Image extracted from [3]), (c) the method of Wang (Image extracted from [28]).

In conclusion, it is necessary to mention that this work could be further improved by finding a theoretical value of the measured threshold for determining quantization precision to ensure a balance with the density of vertices. Thus, one of perspective is to improve the segmentation method using markers to facilitate the grouping of regions by the user.

\section{ACKNOWLEDGMENT}

We thank the anonymous reviewers for their valuable comments which helped improving the quality of the paper. Our implementation is based on the Visualization ToolKit (www.vtk.org).

\section{REFERENCES}

[1] Pierre Alliez and Mathieu Desbrun. Progressive compression for lossless transmission of triangle meshes. In SIGGRAPH'01, pages 195-202, 2001.

[2] M. Attene, S. Katz, M. Mortara, G. Patane, M. Spagnuolo, and A. Tal. Mesh segmentation a comparative study. In SMI6 : Proceedings of the IEEE International Conference on Shape Modeling and Applications,(Washington, DC, USA), page P. 7, 2006.

[3] S. Delest R. Boné and H. Cardot. Hierarchical mesh segmentation using waterfall and dynamics. ISPA: Proceedings of the 5th International Symposium on Image and Signal Processing and Analysis, 2007.

[4] S. Choe, J. Kim, H. Lee, and S. Lee. Random accessible mesh compression using mesh chartication. IEEE Transactions on Visualization and Computer Graphics 15, 2009.

[5] P. Cignoni, F. Ganovelli, E. Gobbetti, F. Marton, F. Ponchio, and R. Scopigno. Bdam batched dynamic adaptive meshes for high performance terrain visualization. EUROGRAPHICS 003, 22(3), 2003.

[6] Paolo Cignoni, Claudio Rocchini, and Roberto Scopigno. Metro: Measuring error on simplified surfaces. Computer Graphics Forum, 17(2):167-174, 1998.

[7] Daniel Cohen-Or, David Levin, and Offir Remez. Progressive compression of arbitrary triangular meshes. In IEEE Visualization, pages 67-72, 1999.

[8] Clement Courbet and Céline Hudelot. Random accessible hierarchical mesh compression for interactive visualization. Eurographics Symposium on Geometry Processing 009, 2009.

[9] S. Delest, B. Romuald, and C. Hubert. Etat de 1 art de la segmentation de maillage 3D par patchs surfaciques. GTMG-2007, Groupe de Travail en Modélisation Géométrique, pages 171-18, mars 2007.

[10] Olivier Devillers and Pierre-Marie Gandoin. Progressive lossless compression of arbitrary simplicial complexes. ACM Transactions on Graphics, 21(Siggraph'2002 Conference proceedings):372-379, 2002.

[11] P.-M. Gandoin and O. Devillers. Compression out-of-core pour la visualisation interactive de maillages. In ACM SIGGRAPH Conference Proc, 2002.

[12] E. Gobbetti and F. Marton. Far voxels : a multiresolution framework for interactive rendering of huge complex $3 \mathrm{~d}$ models on commodity graphics platforms. In In ACM SIGGRAPH ACM Press, 2005.
[13] Hugues Hoppe. Progressive meshes. In SIGGRAPH, pages 99-108, 1996.

[14] Clément Jamin, Pierre-Marie Gandoin, and Samir Akkouche. Compression out-of-core pour la visualisation interactive de maillages. AFIG 008 (Association Française d'Informatique Graphique 008), IRIT Presse, 2008.

[15] Zachi Karni and Craig Gotsman. Spectral compression of mesh geometry. In Proceedings of the 27th annual conference on Computer graphics and interactive techniques, SIGGRAPH'00, pages 279-286, 2000.

[16] Andrei Khodakovsky, Peter Schröder, and Wim Sweldens. Progressive geometry compression. In Proceedings of the 7th annual conference on Computer graphics and interactive techniques, SIGGRAPH '00, pages 271-278, 2000.

[17] Aaron W. F. Lee, Wim Sweldens, Peter Schröder, Lawrence Cowsar, and David Dobkin. Maps: Multiresolution adaptive parameterization of surfaces. In Proceedings of SIGGRAPH8, jul 1998.

[18] H. Lee, G. Lavoué, and F. Dupont. New methods for progressive compression of colored 3d mesh. International Conference on Computer Graphics, Visualization and Computer Vision (WSCG), 2010.

[19] Ho Lee. Compression progressive et tatouage conjoint de maillages surfaciques avec attributs de couleur. Thèse de doctorat, Université Claude Bernard ,Lyon, jun 2011.

[20] Ho Lee, Guillaume Lavoué, and Florent Dupont. Rate-distortion optimization for progressive compression of $3 \mathrm{~d}$ mesh with color attributes. The Visual Computer, 28(2):137-153, 2012.

[21] P. Lindstrom. Out-of-core construction and visualization of multiresolution surfaces. In Sym. on Interactive 3D Graphics, ACM Press, 2003.

[22] Adrien Maglo, Ian Grimstead, and Céline Hudelot. Cluster-based random accessible and progressive lossless compression of colored triangular meshes for interactive visualization. Computer Graphics International 011 (CGI-2011), 2011.

[23] F. Payan. Optimisation de compromis débit-distorsion pour la compression géométrique de maillages surfaciques triangulaires. Thèse de doctorat, STIC de Nice Sophia Antipolis, 2004.

[24] Frédéric Payan and Marc Antonini. An efficient bitallocation for compressing normal meshes with an error-driven quantization. Computer Aided Geometric Design, Special Issue on Geometric Mesh Processing, 22:466-486, Jul 2005.

[25] J. Peng and C. Kuo. Geometry-guided progressive lossless 3d mesh coding with octree (ot) decomposition. ACM Trans. Graph. 24, 5, 2005.

[26] Sébastien Valette, Raphaëlle Chaine, and Rémy Prost. Progressive lossless mesh compression via incremental parametric refinement. Computer Graphics Forum (Proceedings of Symposium on Geometr Processing 2009), (5):1301-1310, Jul 2009.

[27] Sébastien Valette and Rémy Prost. Wavelet-based progressive compression scheme for triangle meshes: Wavemesh. IEEE Trans. Vis. Comput. Graph., 10(2):123-129, 2004.

[28] J. Wang and Y. Zeyun. Surface feature based mesh segmentation. SMI, pages 27, 31 and 35, 2011.

[29] S.-E. Yoon, B. Salomon, R. Gayle, and D. Manocha. Interactive viewdependent rendering of massive models. In Proc. O Visualization,IEEE Computer Society, page 131/138, 2004.

\section{Creative Commons Attribution License 4.0 (Attribution 4.0 International, CC BY 4.0)}

This article is published under the terms of the Creative Commons Attribution License 4.0 https://creativecommons.org/licenses/by/4.0/deed.en_US 